\title{
Clínica y tratamiento de la diabetes tipo 2 en adultos jóvenes en un hospital colombiano
}

\author{
Clinical presentation and treatment of type 2 \\ diabetes in young adults in a Colombian hospital
}

\author{
Diego Julián Falon-Guzmán, Juan Diego Villegas-Suárez, Juliana Cardona-Cardona, \\ Santiago Cardona-Cardona, Sebastián Quintero-López, Juan Daniel Castrillón-Spitia, \\ Gloria María González-Moreno • Pereira (Colombia)
}

DOI: https://doi.org/10.36104/amc.2021.1902

\section{Resumen}

Introducción: la diabetes mellitus tipo 2 (DM2) es el tipo más común de diabetes la cual ocurre generalmente en adultos, sin embargo, hay reportes que la describen en adolescentes y adultos jóvenes.

Objetivo: describir las características clínicas y el tratamiento de la diabetes tipo 2 en adultos jóvenes en un hospital colombiano.

Material y métodos: estudio de corte transversal entre el 2017 y 2019, que incluyó pacientes adultos jóvenes entre 18 y 40 años con DM2, que fueron atendidos en el Hospital Santa Mónica de Dosquebradas, Colombia. La unidad de análisis fueron las historias clínicas. Se incluyeron variables sociodemográficas, clínicas, farmacológicas y control metabólico (HbA1c $<7.0 \%)$. Se realizaron análisis descriptivos y se aplicó una regresión logística binaria $(\mathrm{p}<0.05)$.

Resultados: se identificaron 124 pacientes de los cuales 83 (70.0\%) cumplieron criterios de inclusión, con una edad media de $33.7 \pm 5.3$ años. Unos 28 pacientes eran obesos (33.7\%). Además, 21 pacientes $(25.3 \%)$ presentaron control metabólico. Los fármacos más usados fueron metformina en 64 pacientes $(77.1 \%)$, seguido de las insulinas en 46 pacientes $(55.4 \%)$. Se estableció que 51 de estos (61.4\%) presentaron adherencia al tratamiento. No hubo pacientes con terapia triple como estrategia terapéutica. Los pacientes con retinopatía diabética presentaban una probable asociación con tener control de la enfermedad en el momento del estudio (P=0.048, OR:0.130; IC95\%:0.017-0.987).

Conclusiones: los pacientes adultos jóvenes presentan pobre control metabólico y uso frecuente de insulinas. (Acta Med Colomb 2021; 46. DOI: https://doi.org/10.36104/amc.2021.1902).

Palabras clave: diabetes mellitus tipo 2; adulto joven; insulina; tratamiento farmacológico; cumplimiento y adherencia al tratamiento.

\footnotetext{
Abstract

Introduction: type 2 diabetes mellitus (T2DM) is the most common type of diabetes, generally occurring in adults. However, there are reports which describe it in adolescents and young adults.

Objective: to describe the clinical characteristics and treatment of type 2 diabetes in young adults in a Colombian hospital.

Materials and methods: a cross-sectional study from 2017 to 2019 which included young adult patients between 18 and 40 years old with T2DM who were seen at Hospital Santa Mónica in Dosquebradas, Colombia. The unit of analysis was the medical charts. Sociodemographic, clinical, pharmacological and metabolic control $(\mathrm{HbA} 1 \mathrm{c}<7.0 \%)$ variables were included. Descriptive analyses were performed, and binary logistic regression was applied $(\mathrm{p}<0.05)$.

Results: 124 patients were identified, 83 (70.0\%) of whom met the inclusion criteria, with a mean age of $33.7 \pm 5.3$ years. Some 28 patients were obese (33.7\%). In addition, 21 patients $(25.3 \%)$ had metabolic control. The most frequently used medications were metformin in 64 patients $(77.1 \%)$, followed by insulin in 46 patients (55.4\%). It was determined that 51 of these patients $(61.4 \%)$ were compliant with treatment. No patients received triple therapy as a therapeutic strategy. Patients with
}

Dres. Diego Julián Falon-Guzmán, Juan Diego Villegas-Suarez, Juliana Cardona-Cardona, Santiago Cardona-Cardona, Sebastián QuinteroLópez: Médicos Internos, Fundación Universitaria Autónoma de las Américas, Facultad de Medicina; Dr. Juan Daniel Castrillón-Spitia: Especialista en epidemiología, Fundación Universitaria Autónoma de las Américas, Facultad de Medicina. Grupo de Investigación Biomedicina; Dra. Gloria María Gonzalez-Moreno: Especialista en epidemiología, Hospital Santa Mónica, Dosquebradas. Pereira (Colombia). Correspondencia: Dr. Juan Daniel CastrillónSpitia, Pereira (Colombia).

E-mail: juan.castrillon@uam.edu.co Recibido: 08/VI/2020 Aceptado: 20/IV/2021 
diabetic retinopathy had a probable association with having the disease controlled at the time of the study ( $\mathrm{P}=0.048$, OR:0.130; 95\%CI:0.017-0.987).

Conclusions: young adult patients have poor metabolic control and frequent use of insulins. (Acta Med Colomb 2021; 46. DOI: https://doi.org/10.36104/amc.2021.1902).

Key words: type 2 diabetes mellitus, young adult, insulin, pharmacological treatment, treatment compliance and adherence.

\section{Introducción}

La diabetes mellitus tipo 2 (DM2) es una enfermedad crónica que se produce como consecuencia de un páncreas incapaz de producir suficiente insulina o en la que existe una resistencia periférica a la acción de esta, llevando a aumento de la glucosa en sangre (1). Actualmente la diabetes se puede clasificar como diabetes tipo 1 (DM1), DM2, diabetes gestacional y tipos específicos de diabetes (2). Esta hiperglucemia, lleva a complicaciones en diferentes órganos diana como el corazón, vasos sanguíneos, ojos, riñones entre otros (2). La DM2 es el tipo más común de diabetes la cual ocurre generalmente en adultos; sin embargo, hay reportes que la describen en adolescentes y adultos jóvenes $(3,4)$.

Con base en la información de la International Diabetes Federation (IDF) para el año 2015 se estimaron 415 millones de adultos entre los 20-79 años con DM2 en el mundo, de los cuales 193 millones estaban sin diagnóstico, además, para Sur y Centro América hubo aproximadamente 29.6 millones de pacientes con DM2 y se espera que para el 2040 sean unos 48.8 millones (4). La prevalencia mundial en pacientes con edades entre los 20 y 79 años es $8.8 \%$ y se espera que para el 2040 sea de $10.4 \%$ (4). Para Colombia se estima $8.0 \%$ de prevalencia para DM2, y de este alrededor de $30 \%$ desconoce su enfermedad (4).

En China, con los resultados de una gran encuesta se evidenció que los porcentajes de adultos menores de 40 años con diabetes y prediabetes son del orden de 5.7 y $44.0 \%$ respectivamente (5). Se sabe que la prevalencia global de diabetes mellitus de inicio temprano ha aumentado debido a los estilos de vida sedentarios, la baja actividad física y otros factores no modificables como lo son sexo femenino, edad, antecedentes familiares de la enfermedad, entre otros (5). Además, la evidencia muestra cómo la DM2 de aparición en persona joven tiene un comportamiento más agresivo, lo que lleva al desarrollo prematuro de complicaciones, menor calidad de vida y efectos cardiovasculares a más temprana edad, lo que proyecta a la DM2 en jóvenes como un problema de salud pública $(6,7)$.

En Colombia no se encuentran reportes que caractericen los signos, síntomas, tratamiento y control metabólico de pacientes con DM2 en población joven, por lo cual se planteó caracterizar las variables clínicas y terapéuticas de adultos jóvenes (18-40 años) con DM2 en un hospital de segundo nivel en el municipio de Dosquebradas, Colombia, desde el 2017 hasta el 2019.

\section{Metodología}

Estudio cuantitativo, observacional, descriptivo de corte transversal, que incluyó pacientes adultos jóvenes (entendidos como aquellos con edades entre 18 y 40 años) de cualquier género, con DM2 que fueron atendidos en el Hospital Santa Mónica del municipio de Dosquebradas (Colombia), entre el $1^{\circ}$ de enero de 2017 y el 3 de agosto de 2019. Para ser incluidos los pacientes tenían que pertenecer y estar activos en el programa de control de la DM2 en la institución y contar con al menos una medición de hemoglobina glicosilada (HbA1c). Los encargados de la atención de los pacientes del programa de DM2 son médicos generales con dedicación exclusiva a este, los cuales abarcan a la totalidad de pacientes con diagnóstico confirmado de la enfermedad, independientemente de su estado metabólico.

Los criterios de exclusión fueron pacientes a quienes no se definía claramente en la historia clínica el diagnóstico de DM2 o quienes presentaran diabetes tipo 1, además, las mujeres embarazadas diagnosticadas con diabetes gestacional.

La unidad de análisis fueron las historias clínicas de los pacientes, de las cuales se obtuvieron los datos. Se creó un instrumento de recolección de información en el programa Epi Info 7.0 con opciones únicas de elección para limitar los sesgos de información. Las variables incluidas en el estudio fueron:

- Sociodemográficas: sexo, nivel educativo, edad (en años).

- Clínicas: obesidad, hemoglobina glicosilada (HbA1C), comorbilidades, complicaciones de la DM2 (descripción en la historia clínica de la presencia o no del diagnóstico confirmado de complicaciones secundarias a la enfermedad).

- Tratamiento: antidiabéticos orales (nombre, dosis en $\mathrm{mg} /$ día), insulinas (nombre de insulina, dosis en UI/día), sulfonilureas (nombre, dosis en mg/día), inhibidores de la DPP4 (nombre, dosis en mg/día), análogos de la GLP-1 (nombre, dosis en $\mu \mathrm{g} /$ día).

Se consideró como paciente en control metabólico a aquel con HbA1c menor de $7.0 \mathrm{mg} / \mathrm{dL}$. Además, se consideró paciente adherente al tratamiento aquel cuyo médico explícitamente consignó en la historia clínica si había o no adherencia al tratamiento según los criterios de Morisky Green (8), los cuales evalúan el cumplimiento terapéutico de la medicación. 
TRABAJOS ORIGINALES • Diabetes tipo 2 en adultos jóvenes

Para el análisis de los datos se utilizó el programa SPSS versión 23 para Windows (IBM, USA). Se empleó estadística descriptiva como promedio, desviación estándar, valores mínimos y máximos, intervalos de confianza para variables continuas y porcentajes para variables categóricas. Se realizaron pruebas X2 para la comparación de variables categóricas; se incluyeron los valores de p, los OR y los intervalos de confianza. Se aplicó un modelo de regresión logística binaria usando como variable dependiente si el paciente estaba en control metabólico (sí/no), y como variables independientes aquellas que se asociaron de manera estadísticamente significativa en los análisis bivariados. Se determinó como nivel de significancia estadística una $\mathrm{p}<0.05$. Con relación al sesgo de información y de confusión se estableció una p menor de 0.05 siendo más estrictos que el criterio de Hosmer-Lemeshow para reducir la sobreestimación de los resultados y se usó un análisis apropiado para variables categóricas.

Esta investigación fue avalada por el Comité de Ética en Investigación de la Fundación Universitaria Autónoma de las Américas en la categoría de investigación sin riesgo, al igual que se contó con la aprobación del Comité Científico y de Seguridad del Paciente del Hospital. Asimismo, se respetaron los principios establecidos por la Declaración de Helsinki (9).

\section{Resultados}

Se encontraron 124 pacientes durante el periodo de estudio, de los cuales 83 pacientes $(70.0 \%)$ cumplieron criterios de inclusión. Se presentaron 59 mujeres (71.1\%). Cincuenta y siete pacientes se ubican en el grupo de edad entre 31 y 40 años $(68.7 \%)$. En la Tabla 1 se describen las características sociodemográficas de la población.

\section{Características clínicas}

Se presentaron 11 pacientes $(13.3 \%)$ con nefropatía diabética, además, cinco pacientes presentaron retinopatía diabética (6.0\%), tres casos neuropatía diabética (3.6\%) y a dos pacientes se les reportó pie diabético (2.4\%). En la primera consulta de control a 21 pacientes $(25.3 \%)$ se les reportó una $\mathrm{HbA} 1 \mathrm{c}$ menor de $7.0 \%$, mientras que en la segunda consulta 45 pacientes tenían reportada la HbA1c (52.9\%) y de éstos 17 presentaron control metabólico (37.7\%). En la Tabla 2 se describen las principales características clínicas de la población.

\section{Tratamiento}

Frente a la adherencia descrita por parte de los médicos al tratamiento instaurado a cada paciente, se reportó que fueron adherentes 51 de éstos (61.4\%). En las historias clínicas de 73 pacientes $(87.9 \%)$ se encontraron recomendaciones para realizar cambios a estilos de vidas saludables como parte del tratamiento no farmacológico. También en el tratamiento se encontró el uso concomitante de biguanidas con insulinas en 34 pacientes $(40.9 \%)$, biguanidas con sulfonilureas en cuatro
Tabla 1. Características sociodemográficas de 83 pacientes menores de 40 años con DM2.

\begin{tabular}{|c|c|c|}
\hline \multicolumn{1}{|c|}{ Variables } & Frecuencia & Porcentaje \\
\hline Sexo & 24 & 28.9 \\
\hline Hombre & 23 & 27.7 \\
\hline Nivel educativo & 45 & 54.2 \\
\hline Sin estudios & 4 & 4.8 \\
\hline Primaria & 3 & 3.6 \\
\hline Bachiller & 8 & 9.6 \\
\hline Técnico & $33.7 / 5.3$ & \\
\hline Universitario & 16 & 19.2 \\
\hline Edad (media/DE) años & & \\
\hline Tabaquismo & \multicolumn{2}{l}{} \\
\hline DE: desviación estándar. & & \\
\hline
\end{tabular}

Tabla 2. Características clínicas de 83 pacientes menores de 40 años con DM2.

\begin{tabular}{|c|c|c|}
\hline Variable & $\mathbf{N}$ & $\%$ \\
\hline \multicolumn{3}{|l|}{ Obesidad } \\
\hline $\mathrm{IMC}>30$ & 28 & 33.7 \\
\hline IMC (media/DE) Kg/m² & $28.0 / 6.3$ & \\
\hline HbAlc (media/DE) \% & $8.9 / 2.9$ & \\
\hline $\mathrm{HbA} 1 \mathrm{c}$ Control (media/DE) \% & $8.6 / 2.6$ & \\
\hline \multicolumn{3}{|l|}{ Comorbilidades } \\
\hline Hipertensión & 36 & 43.4 \\
\hline Dislipidemia & 18 & 21.7 \\
\hline Hipotiroidismo & 15 & 18.1 \\
\hline Enfermedad coronaria & 5 & 6.0 \\
\hline Accidente cerebrovascular & 1 & 1.2 \\
\hline Otros & 11 & 13.2 \\
\hline \multicolumn{3}{|c|}{$\begin{array}{l}N \text { : número observacional. IMC: índice de masa corporal. DE: desviación estánda } \\
K G \text { : Kilogramo. } M^{2} \text { : metro cuadrado. HbAlc: hemoglobina glicosilada. }\end{array}$} \\
\hline
\end{tabular}

pacientes (4.8\%), biguanidas con análogos de la GLP-1 o inhibidores de la DPP4 lo presentaban uno y dos pacientes respectivamente (1.2 y $2.4 \%)$; además, hubo cuatro casos de uso concomitante entre insulinas con sulfonilureas (4.8\%). No hubo pacientes con terapia triple como estrategia terapéutica. En la Tabla 3 se describe el uso de fármacos para el tratamiento de la DM2.

Adicionalmente, aquellos pacientes con $\mathrm{HbA} 1 \mathrm{c}>9 \%$, era más probable que se les formulara una insulina $(\mathrm{p}=0.001$; OR:5.571, IC:95\%: 2.033-15.271), tanto de larga acción (P=0.002; OR:4.083, IC:95\%: 1.608-10.372) como una de corta acción ( $\mathrm{P}=0.009$; OR:3.415, IC:95\%: 1.345-8.675). No hubo asociación entre el uso o no de los hipoglicemiantes orales y el nivel de $\mathrm{HbA} 1 \mathrm{c}$ de los pacientes.

\section{Multivariado}

Al realizar una regresión logística binaria con presentar o no control metabólico con aquellas variables asociadas 
Tabla 3. Fármacos usados para el tratamiento de 83 pacientes menores de 40 años con DM2.

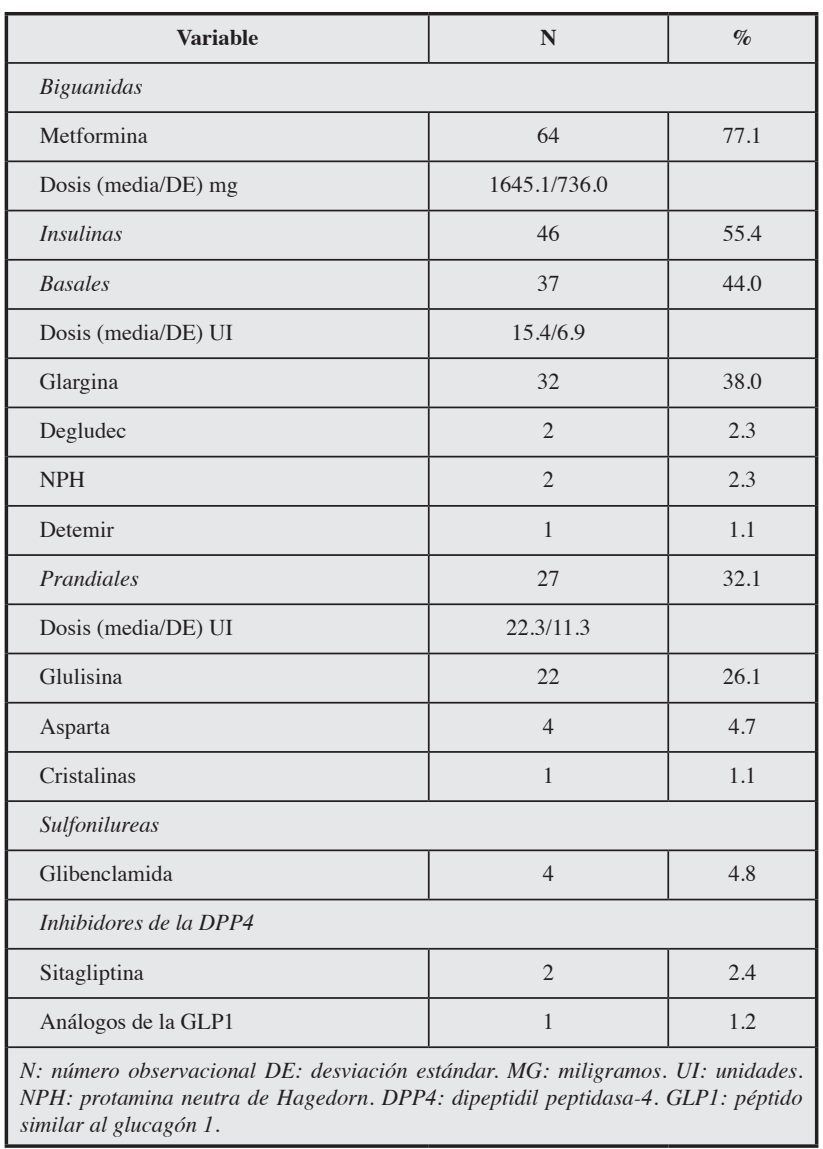

significativamente en los análisis bivariados, se encontró que los pacientes con retinopatía diabética presentaban una probable asociación con tener control de la enfermedad en el momento del estudio. En la Tabla 4 se describen los resultados de la regresión logística binaria.

\section{Discusión}

Con el presente estudio se documentaron las características clínicas y tratamiento prescrito en adultos jóvenes con DM2 en una institución de segundo nivel en Colombia. Estos datos pueden orientar mejor sobre las posibles fallas que se están dando en el tratamiento médico en esta población, evidenciando para este grupo de pacientes un posible enfoque que difiere del recomendado por las guías de práctica clínica.

Como se ha reportado en la literatura ser mujer, en especial ser paciente joven, se considera un factor de riesgo para desarrollar DM2 (10). Un estudio reportó que para esta población dicha patología oscila entre 60 y $80 \%$ (11), datos similares a los descritos en el presente estudio. Esto debe llevar a replantear estrategias de identificación temprana de mujeres con alto riesgo de diabetes; una de estas puede ser la búsqueda activa de la prediabetes en mujeres jóvenes.

También es conocido que entre los factores de riesgo identificados para el desarrollo de la DM2 están la obesidad, el tabaquismo, el síndrome metabólico y la hipertensión
Tabla 4. Regresión logística binaria para la variable presentar control metabólico.

\begin{tabular}{|l|c|c|c|c|}
\hline \multirow{2}{*}{ Variable } & P & \multirow{2}{*}{ OR } & \multicolumn{2}{c|}{ 95\% I.C. } \\
\cline { 4 - 6 } & & & Inferior & Superior \\
\hline Sexo & 0.070 & 0.350 & 0.112 & 1.089 \\
\hline Adherente al tratamiento & 0.078 & 0.309 & 0.084 & 1.140 \\
\hline Retinopatía & 0.048 & 0.130 & 0.017 & 0.987 \\
\hline Insulinas & 0.090 & 2.669 & 0.858 & 8.298 \\
\hline Constante & 0.200 & 3.868 & & \\
\hline P: valor de probabilidad. OR: odds ratio. I.C: intervalo de confianza. \\
\hline
\end{tabular}

arterial (12). Estos fueron frecuentemente encontrados en la población del presente reporte; para el caso el tabaquismo la proporción de pacientes fumadores supera la nacional $($ Colombia $=12.8 \%)$ e inclusive es mayor al del departamento (Risaralda $=17.3 \%)(13)$. La proporción de pacientes con hipertensión arterial es superior a la reportada en otras poblaciones, inclusive en ciudades vecinas, en estudios elaborados en la población general (14).

Lo anterior permite observar que los pacientes del estudio presentan factores de riesgo que deben ser intervenidos buscando no sólo el control de la DM2, sino la reducción de las complicaciones asociadas a ésta. Cabe recordar a los profesionales de la salud que el primer paso en el tratamiento de la DM2 son los cambios de estilo de vida, frecuentes en el presente reporte, por lo que la búsqueda de las razones que expliquen el pobre control metabólico y el uso frecuente de insulinas deben explorarse en futuros estudios, incluyendo el seguimiento a la adherencia de estas medidas no farmacológicas.

En el caso de la obesidad, la proporción encontrada es mayor al de la población general colombiana (16.5\%) (15), ya que uno de cada tres pacientes presentaba obesidad, lo que suma elementos que describen una población con múltiples factores de riesgo para la enfermedad. En los pacientes jóvenes los estilos de vida poco saludables, la poca adherencia o una respuesta diferente al tratamiento adecuado, pueden ser razones que lleven a un uso frecuente de insulinas, lo cual se relaciona con la aparición más temprana de complicaciones (16). Los cambios en el estilo de vida son la principal característica para tratar y para lograr mejores resultados a largo plazo en esta población (10).

Adicionalmente, es importante mencionar que el concepto de síndrome metabólico aplicado en los adultos (agrupación de al menos tres factores de riesgo: obesidad, hiperglucemia, dislipidemia e HTA, elementos asociados a insulinorresistencia), no está completamente adaptado en personas jóvenes, en especial en niños, lo que resume en una falta de consenso frente a la definición en esta población (17). Sin embargo, la identificación de factores de riesgo para estas patologías incluidas en el síndrome metabólico es adecuada a tempranas edades, conservando los factores de 
riesgo descritos en el adulto con variaciones en sus puntos de corte $(18,19)$. Además, existen herramientas de tamizaje ya validados en Colombia como el FINDRISC, el cual incluye diferentes parámetros como el perímetro abdominal, el IMC, entre otros, que valoran la probabilidad de desarrollar DM2 y en el que, de identificar el riesgo, las intervenciones no farmacológicas juegan un papel prioritario (20). Sin embargo, es importante recordar que esta herramienta fue validad en personas mayores de 18 años.

Se aprecia cómo en Villavicencio, una ciudad colombiana, tienen pacientes menores de 50 años con una mayor proporción en control metabólico $(55.8 \%)$ en comparación con la población del presente estudio (21). Sin embargo, un reporte en Asia muestra porcentajes similares (22). Es importante mencionar que la generación de información sobre el control metabólico en la población menor de 40 años es escasa; se deben identificar las particularidades del control de esta enfermedad en estos grupos de edad para desarrollar estrategias tanto diagnósticas como terapéuticas que permitan llegar al control metabólico.

El uso de insulinas es recomendado como uno de los últimos eslabones en el tratamiento de DM2 al estar comprometida la función de las células beta del páncreas, aunque en los últimos consensos realizados por Asociación Americana de Endocrinólogos Clínicos, Colegio Americano de Endocrinología, Asociación Americana de la Diabetes y Asociación Europea para el consenso del Estudio de la Diabetes, refieren que está indicado como primera línea si se tiene una $\mathrm{HbA} 1 \mathrm{c} \geq 9 \%$, ya que algunos estudios han demostrado que la terapia intensiva de insulina en DM2 a corto plazo en paciente con diagnóstico reciente y $\mathrm{HbA} 1 \mathrm{c}$ $\geq 9 \%$ tiene efectos positivos sobre los mecanismos fisiopatológicos de la misma (23). Lo anterior puede justificar el elevado uso de insulina en la población del presente estudio como se observó en aquellos pacientes con $\mathrm{HbA} 1 \mathrm{c}>9 \%$; sin embargo, esto debe ser individualizado e iniciar la terapia con hipoglucemiantes orales sobre la insulinización (20).

Si bien, según diversas guías de manejo de la DM2 indican que el tratamiento en estos pacientes puede ser con metformina e insulinas, las guías de la Asociación Americana de Diabetes recomiendan el uso de insulinas como primera línea en paciente con una $\mathrm{HbA} 1 \mathrm{c}>10 \%$, pacientes con terapia triple sin control metabólico, pacientes con catabolismo aumentado, cetoacidosis o en su defecto quienes presenten variantes de la enfermedad mixta DM1 y DM2, entre otros, pero es claro que no se incluye la edad como un factor para el inicio de insulina (24).

Además de lo anterior, llama la atención la mayor prescripción de insulinas análogas sobre las convencionales, conducta que se justifica cuando se presentan reacciones adversas con las insulinas convencionales, tales como hipoglucemia o falta de control de la glucemia (entre otras) con el uso de las convencionales, ya que fuera de estas consideraciones no hay evidencia que demuestre mayor eficacia o costo efectividad de las análogas sobre éstas. (25 - 31).
Así mismo, se debe recordar que las sulfonilureas en los últimos años, en especial la glibenclamida, han sido relegadas por otros antidiabéticos a fármacos de tercera línea $(2,20)$. Si bien las sulfonilureas tienen un buen impacto en la reducción de la $\mathrm{HbA} 1 \mathrm{c}(1.5 \% 2 \%)$, éstas presentan múltiples reacciones adversas como la hipoglucemia, además de la descripción de impactos importantes en el músculo liso y en el miocardio (receptores 2A y 2B de las sulfunilureas), siendo además contraindicadas en la presencia de enfermedad renal (32, 33). En caso de utilizar este grupo farmacológico, se debe usar la glimepirida o la glicazida por la menor frecuencia de reacciones adversas de estos fármacos y por las diferencias en la farmacodinamia frente a la glibenclamida $(2,20)$.

Entre las limitaciones del estudio se encuentra que la información proviene de registros clínicos que pueden contener errores de redacción o expresiones que no permitan la adecuada interpretación de la intención del médico tratante, así como no se logró definir quién realizó el diagnóstico de las complicaciones derivadas de la DM2, las cuales estaban descritas en las historias clínicas. Además, se debe considerar que no se tuvo en cuenta como criterio de inclusión una segunda HbA1c, por lo cual no fue posible evaluar en toda la población el cambio de ésta entre consultas o el posible impacto de las terapias prescritas. También se debe mencionar el número de pacientes incluidos el cual es pequeño y limita el alcance y generalización de los resultados; sin embargo, después de la búsqueda bibliográfica realizada, se puede afirmar que es el primer estudio que caracteriza a población menor de 40 años con DM2 en Colombia con una observación retrospectiva de dos años, lo cual es una fortaleza del presente reporte.

Como conclusión se describe que los pacientes menores de 40 años con DM2 presentan pobre control metabólico, escasa formulación de medidas no farmacológicas y un uso frecuente de insulinas a pesar de que su tratamiento no difiere a la de la población de mayor edad con base en las recomendaciones de diferentes guías clínicas. Nuevos estudios deben ser desarrollados en la población colombiana joven explorando las diferencias entre los tratamientos disponibles y el impacto que éste pueda tener en el control metabólico y en el pronóstico de un paciente.

\section{Agradecimientos}

Se agradece a los profesores Andrés Gaviria Mendoza, Paula Andrea Moreno y a la profesora Alexandra Agudelo Ramírez por sus aportes realizados al presente manuscrito.

\section{Referencias}

1. Organización Mundial de la Salud. Definition, diagnosis and classification of diabetes mellitus and its complications. OMS [Internet]. 1999 [citado el 27 de febrero de 2020]: 1-66. Disponible en: https://apps.who.int/iris/bitstream/ handle/10665/66040/WHO_NCD_NCS_99.2.pdf? sequence=1\&isAllowed=y

2. American Diabetes Association. Standards of Medical Care in diabetes. ADA [Internet]. 2020 [citado el 27 de febrero de 2018];43(1):1-2. Disponible en:https:// care.diabetesjournals.org/content/diacare/43/Supplement_1/S1.full.pdf

3. Gómez-Huelgas R. Ensayo clínico para mantener el control glucémico en jóvenes con diabetes tipo 2. Rev Clin Esp. 2012;212(10):500. doi: 10.1016/j. rce.2012.07.015 
4. International Diabetes Federation. Atlas de la Diabetes de la IDF.IDF [Internet] .2019 [ citado el 27 de febrero de 2018]:1-180. Disponible en: https://diabetesatlas. org/upload/resources/material/20200302_133352_2406-IDF-ATLAS-SPANBOOK.pdf

5. Pan J, Jia W. Early-onset diabetes: an epidemic in China. Front Med. 2018;12(6):624-633. doi: 10.1007/s11684-018-0669-1.

6. Lascar N, Brown J, Pattison H, Barnett AH, Bailey CJ, Bellary S. Type 2 diabetes in adolescents and young adults. Lancet Diabetes Endocrinol. 2018;6(1):69-80. doi: 10.1016/S2213-8587(17)30186-9.

7. Nadeau KJ, Anderson BJ, Berg EG, Chiang JL, Chou H, Copeland KC, et al. Youth-Onset Type 2 Diabetes Consensus Report: Current Status, Challenges, and Priorities. Diabetes Care. 2016;39(9):1635-42. doi: 10.2337/dc16-1066.

8. Morisky, D, Green L, Levine D. Concurrent and Predictive Validity of a Selfreported Measure of Medication Adherence. Medical Care. 1986;24: 67-74.

9. World Medical Association. Ethical Principles for Medical Research Involving Human Subjects. WMA [Internet]. 2013 [citado el 23 de agosto de 2020];310(20):1-4. Disponible en: https://www.wma.net/wp-content/uploads/2016/11/DoH-Oct2013-JAMA.pdf

10. Magliano DJ, Sacre JW, Harding JL, Gregg EW, Zimmet PZ, Shaw JE. Young-onset type 2 diabetes mellitus - implications for morbidity and mortality. Nat Rev Endocrinol. 2020:1-11. doi: 10.1038/s41574-020-0334-Z.

11. Khanolkar AR, Amin R, Taylor-Robinson D, Viner R, Warner J, Stephenson T. Ethnic Minorities Are at Greater Risk for Childhood-Onset Type 2 Diabetes and Poorer Glycemic Control in England and Wales. Journal of Adolescent Health. 2016;59(3):354-361. doi: 10.1016/j

12. Kyrou L, Tsigos C, Mavrogianni C, Cardon G, Stappen VV, Latomme J, et al. Sociodemographic and lifestyle-related risk factors for identifying vulnerable groups for type 2 diabetes: a narrative review with emphasis on data from Europe. BMC Endocrine Disorders. 2020;20(1):134. doi: 10.1186/s12902-019-0463-3

13. Ministerio de Salud y Protección Social de Colombia. Socialización del informe final de evaluación de necesidades para la ampliación del Convenio Marco de Control del Tabaco Cifras Oficiales para Colombia. MinSalud [Internet]. 2007 [ citado el 02 de abril de 2020]:1-3. Disponible en: https://www.minsalud.gov.co/ Documents/General/Cifras-tabaco-Colombia.pdf

14. Castrillón-Spitia JD, Franco-Hurtado A, Garrido-Hernández C, JaramilloPatiño J, Londoño-Moncada MA, Machado-Alba JE. Utilización de fármacos antihipertensivos, efectividad e inercia clínica en pacientes. Rev. Colomb. Cardiol. 2018;25(4):249-256. doi: 10.1016/j.rccar.2017.08.027

15. Ministerio de Salud y Protección Social. Guía de práctica clínica para la prevención, diagnóstico y tratamiento del sobrepeso y la obesidad en adultos. MinSalud [Internet]. 2016 [citado el 03 de abril de 2020]:1-1020. Disponible en: http://gpc.minsalud.gov.co/gpc_sites/Repositorio/Conv_637/GPC_obesidad/ GUIA_SOBREPESO_OBESIDAD_ADULTOS_COMPLETA.pdf.

16. Hernández-Ruiz de Eguilaz M, Batlle M, Martínez de Morentin B, San-Cristóbal R,Pérez-Díez S, Navas-Carretero S, et al. Cambios alimentarios y de estilo de vida como estrategia en la prevención del síndrome metabólico y la diabetes mellitus tipo 2: hitos y perspectivas. An. Sist. Sanit. Navar. 2016;39(2):269-289.

17. Magge SN, Goodman E, Armstrong SC. The metabolic syndrome in children and adolescents: shifting the focus to cardiometabolic risk factor clustering. Pediatrics 2017;140, e20171603.

18. De Filippo G. Obesidad y síndrome metabólico. EMC Pediatría. 2021; 56 (1):1-7, doi.org/10.1016/S1245-1789(21)44717-7
19. Perreult L. Obesity in adults: Prevalence, screening, and evaluation [internet]: Pi- Sunyer X;2021 [consultado 27 marzo de 2021]. Disponible en: https:/ www.uptodate.com/contents/obesity-in-adults-prevalence-screening-and-ev aluation? search $=$ obesity \& source $=$ search_result $\&$ selectedTitle $=1 \sim 150 \&$ usa ge_type $=$ default $\&$ display_rank $=1$

20. Ministerio de Salud y Protección Social de Colombia. Guía de práctica clínica para el diagnóstico, tratamiento y seguimiento de la diabetes mellitus tipo 2 en la población mayor de 18 años. MinSalud [Internet]. 2016 [ citado el 04 de abril de 2020]:1-606. Disponible en: http://gpc.minsalud.gov.co/gpc_sites/Repositorio/ Conv_637/GPC_diabetes/DIABETES_TIPO_2_COMPLETA.pdf.

21. Piñeros-Garzón FS, Rodríguez-Hernández JM. Factores de riesgo asociados al control glucémico y síndrome metabólico en pacientes con diabetes mellitus tipo 2. Villavicencio, Colombia. Univ. Salud. 2019;21(1):61-71. Doi: 10.22267/ rus. 192101.140

22. Unnikrishnan R, Anjana RM, Anandakumar A, Harish R, Saravanan J, Ali MK, et al. Younger-onset versus older-onset type 2 diabetes: Clinical profile and complications. J Diabetes Complications. 2017;31 (6):971-975. doi: 10.1016/j. jdiacomp.2017.03.007 j.

23. Weng J. Short-term intensive insulin therapy could be the preferred option for new onset Type 2 diabetes mellitus patients with HbA1c $>9$. J Diabetes. 2017;9(10):890-893. doi: 10.1111/1753-0407.12581.

24. Castillo-Nuñez Y,Aguilar-Salinas CA.Asociación Latinoamericana de Diabetes. Análogos de insulina: el dilema entre el costo y la efectividad. Revista de la ALAD [Internet]. 2012 [citado el 27 de febrero de 2020];2(2):236-273. Disponible en: http://www.revistaalad.com/pdfs/Revista-de-la-Alad-V2N2.pdf\#page=13

25. Luo J, Khan NF, Manetti T, Rose J, Kaloghlian A, Gadhe B, et al. implementation of a Health Plan Program for Switching from Analogue to Human Insulin and Glycemic Control Among Medicare Beneficiaries With Type 2 Diabetes. JAMA 2019; 321:374-384. doi: 10.1001/jama.2018.21364.

26. Lipska KJ. Insulin Analogues for Type 2 Diabetes. JAMA. 2019;321(4):350-351 doi:10.1001/jama.2018.21356

27. Calero-Bernal M, Varela-Aguilar J. Diabetes tipo 2 infantojuvenil. Revista clínica española. 2018; 218(7): 372-38. doi: 10.1016/j.rce.2018.03.020

28. Yamada T, Kamata R, Ishinohachi K, Shojima N, Ananiadou S, Nom H, et al. Biosimilar vs originator insulins: Systematic review and meta-analysis. Diabetes Obes Metab. 2018; 20(7):1787-1792. doi: 10.1111/dom.13291.

29. Shafie AA, Ng CH, Tan YP, Chaiyakunapruk N. Systematic Review of the Cost Effectiveness of Insulin Analogues in Type 1 and Type 2 Diabetes Mellitus. Pharmacoeconomics. 2017 ;35(2):141-162. doi: 10.1007/s40273-016-0456-2.

30.Semlitsch T, Engler J, Siebenhofer A, Jeitler K, Berghold A, Horvath K. (Ultra-)long-acting insulin analogues versus NPH insulin (human isophane insulin) for adults with type 2 diabetes mellitus. Cochrane Database Syst Rev. 2020; 11:CD005613. doi: 10.1002/14651858.CD005613.pub4.

31. Madenidou AV, Paschos P, Karagiannis T, Katsoula A, Athanasiadou E, Kitsios K, et al. Comparative Benefits and Harms of Basal Insulin Analogues for Type 2 Diabetes: A Systematic Review and Network Meta-analysis. Ann Intern Med. 2018; 169(3):165-174. doi: 10.7326/M18-0443.

32. Sánchez, L.P. Sulfonilureas en el tratamiento del paciente con diabetes mellitus tipo 2. Endocrinología y Nutrición. 2008; 55(2):17-25. doi: 10.1016/S15750922(08)76259-4

33. Llinás Castro R,Alvis Estrada L, Mendoza Goez L. Evaluación de la prescripción de glibenclamida en diabéticos tipo 2. Salud UIS 49(1):9-15 doi: 10.18273/ revsal.v49n1-2017001. 